\title{
Nitrogen enrichment causes thermal acclimation of soil microbial respiration
}

\author{
Huimin Sun ${ }^{1}$, Hongyang Chen ${ }^{2}$, Jintao $\mathrm{Li}^{1}$, Yan Zhang ${ }^{3}$, Xiang $\mathrm{Liu}^{4}$, Bo LI ${ }^{2}$, Shurong \\ Zhou ${ }^{5}$, and Ming $\mathrm{Nie}^{1}$ \\ ${ }^{1}$ Ministry of Education Key Laboratory for Biodiversity Science and Ecological \\ Engineering, Institute of Biodiversity Science, Fudan University, Shanghai 200438, China \\ ${ }^{2}$ Fudan University \\ ${ }^{3}$ Ministry of Education Key Laboratory for Biodiversity Science and Ecological \\ Engineering, Coastal Ecosystems Research Station of the Yangtze River Estuary, School of \\ Life Sciences, Fudan University \\ ${ }^{4}$ Lanzhou University \\ ${ }^{5}$ Hainan University
}

November 2, 2021

\begin{abstract}
The feedback between soil carbon $(\mathrm{C})$ and climate has the potential to decrease in magnitude over time due to the thermal acclimation of microbial respiration, while, whose strength is highly uncertain, partly because the response of microbial respiration is regulated by multiple environmental factors simultaneously rather than by temperature alone. Using a 9-year two-way factorial experiment involving warming and multilevel nitrogen enrichment treatments from an alpine grassland, we show that microbial respiration acclimates to warming only under nitrogen enrichment and that the strength of thermal acclimation increases as nitrogen enrichment. We identified two contrasting pathways - via an enhancement of acclimation by soil acidification and a weakening of acclimation by the inhibition of soil $\mathrm{C}$ availability and stimulation of soil C-degrading enzymes - with a net positive effect of nitrogen enrichment on microbial thermal acclimation. Our findings emphasize the importance of considering multiple environmental factors in shaping the strength of thermal acclimation.
\end{abstract}

\section{Hosted file}

EL-MainText.docx available at https://authorea.com/users/444160/articles/543965-nitrogenenrichment-causes-thermal-acclimation-of-soil-microbial-respiration 